\title{
Role of magnetic resonance imaging on fingers in diagnosis of post-traumatic tendon and ligament injuries
}

\author{
Hayam Abdelmonsif Abdellatif ${ }^{1}$, Mohammed Abd El Aziz Maaly ${ }^{3}$, Belal Said Hefny Ibrahim Soltan²*(10 and \\ Mohammed Shawky Abduallah²
}

\begin{abstract}
Background: Magnetic resonance (MR) imaging is a powerful method for evaluating acute and chronic lesions of the stabilizing articular elements (volar plate and collateral ligaments) of the fingers and thumbs, the condition of tendons, the presence of a tear, the number of affected tendons, the extent of tendon retraction, and the presence of associated lesions. This study was done to clarify the value of magnetic resonance imaging (MRI) in assessment of trauma-related injuries of the tendons and ligaments of the fingers and subsequently positively affect the surgical decisions in such cases.
\end{abstract}

Results: This study included 42 patients (33 males and 9 females) with a mean age of 35 years. All of them had history of trauma. Male affection was $78.5 \%$, while females constituted only $21.5 \%$, striking right-sided-hand affection (92.9\%). The most affected finger was the thumb. The most common affected sites were extending from the metacarpophalangeal (MCP) till the proximal interphalangeal (PIP). The tendons were affected in 42.9\%; meanwhile, ligamentous affection represented $57.2 \%$. The flexor tendon tear represented by $28.6 \% \%$, while extensor tendon tear occurred in $14.3 \%$. The study also showed that partial thickness tear is represented by $66.7 \%$, while complete thickness tear occurred in about 33.3\%. Bone marrow edema and post-traumatic tenosynovitis occurred in about 14\% of cases.

Conclusions: Magnetic resonance imaging is an essential technique to obtain a correct pre-surgical diagnosis. It is of utmost importance to possess an in-depth knowledge of finger radiological anatomy in detail, as well as the appearance of the different pathologic entities on MRI. It provides a great assessment of the tendons and ligaments tear by whether partial or complete, any associated marrow edema or bony fragment avulsion and the extent of retraction in cases of complete tear.

Keywords: Hand, Finger, Tendon, Ligament, Injury, Tear, Magnetic resonance imaging

\section{Background}

Magnetic resonance imaging is a powerful method for evaluating acute and chronic lesions of the stabilizing articular elements (volar plate and collateral ligaments) of the fingers and thumbs, particularly in the frequently affected proximal interphalangeal and

\footnotetext{
*Correspondence: belal.soultan@med.menofia.edu.eg

2 Faculty of Medicine, Menoufia University, shebin el kom, Menoufia

Governorate, Egypt

Full list of author information is available at the end of the article
}

metacarpophalangeal joints. Radiologists need to understand the full spectrum of finger abnormalities and associated MR imaging findings [1].

Injuries to the tendons of the hand and wrist occur commonly. Inflammation of the tendon (tendinitis), tendon sheath (tenosynovitis), or perivascular bundle (peritendinitis) may occur alone or in combination. Tendinosis is a degenerative process seen in overuse and aging that results in mucoid degeneration, vascular ingrowth, and cartilage metaplasia. Tendon ruptures may be partial or complete. Tendon subluxations and 
dislocations also occur, typically in patients with inflammatory arthropathies such as rheumatoid arthritis [2].

Magnetic resonance imaging allows optimal assessment of the condition of tendons, thus making it possible to evaluate the presence of a tear, the number of affected tendons, the extent of tendon retraction, and the presence of associated lesions. This information is used to determine the correct surgical plan and surgical approach and is especially useful for closed fractures [3].

The extensor hood of the metacarpophalangeal (MCP) joint can also be injured. The extensor digitorum communis tendon is joined by the sagittal bands, which serve as stabilizers of the extensor tendon and form one of the main components of the extensor hood. These sagittal bands attach to the extensor tendon dorsally and to the volar plate and the transverse intermetacarpal ligament on the volar side of the MCP [4].

Injuries to the extensor mechanism of the finger are common because it consists of thin, superficially located structures. These anatomic structures predispose tendons to lacerations and to closed tendon injuries, including avulsion [5].

As with the extensor tendon apparatus, a division of the flexor tendons into multiple zones has been established based on the distinct anatomic differences responsible for different prognoses of otherwise identical tendon injuries [6].

Magnetic resonance imaging criteria for diagnosis of acute collateral ligament tears include discontinuity, detachment, or thickening of the ligament together with increased intraligamentous signal intensity on T2-weighted images, which is indicative of edema or hemorrhage. Obliteration of the fat planes around the ligament and extravasation of joint fluid into the adjacent soft tissues may also be observed. Chronic tears often demonstrate thickening of the ligament, which is probably secondary to scar formation. Thinning, elongation, or a wavy contour of the ligament may also be seen [7].

Lesions of the pulley system are recognized with increasing frequency due to the growing popularity of activities, such as rock climbing, that impose extensive stress on the supporting structures of the hand and fingers [8].

Lesions of the pulley system can be diagnosed indirectly by detection of a gap between the flexor tendon and the bone on sagittal images obtained during forced flexion, a finding referred to as the "bowstringing sign" [9].

A trigger finger is a common condition and is generally diagnosed clinically. It is either blockage or triggering of the finger from flexion to extension and in most cases involves the MCP joint. The patho-physiologic mechanism of trigger fingers is controversial [10].
Magnetic resonance imaging demonstrates findings of thickening and inflammation of the annular pulley 1 (A1 pulley), and post-gadolinium contrast images may demonstrate pulley enhancement [11].

The aim of this study was to clarify the value of MRI in assessment of trauma-related injuries of the tendons and ligaments of the fingers and subsequently positively affect the surgical decisions in such cases.

\section{Methods}

This prospective study was performed in Radiology Department of our institution, during the period from August 2018 to August 2019. This study included 42 patients, 33 males and 9 females, with age range from 11 to 56 years (mean age 35 years). All of them had history of trauma.

\section{Inclusion criteria}

Patients presented with swelling, limitation of function or pain following history of trauma.

\section{Exclusion criteria}

The excluded cases included those presented with simple post-traumatic fractures without suspected soft tissue injuries. Also, patients with rheumatoid arthritis were excluded either by their clinical history or by laboratory investigations as positive rheumatoid factor (RF) and anti-cyclic citrullinated peptide (anti-CCP). Patients with history of related operations or internal fixations were excluded. Finally, patients with cardiac pacemaker or critically located foreign body were not subjected to MRI examination.

\section{Ethical considerations}

All study procedures were carried out and approved by the ethical committee of our institution. All participants in this study received a detailed explanation about the aim, objectives, and methodology of the study before enrollment. All subjects included in the current research gave their written informed consent to publish the data contained within the study.

\section{All patients were subjected to the following History taking \\ Clinical examination General examination for systemic disease. \\ Local examination of the fingers.}

Technique of MRI study of the fingers as follows MRI was performed using 1.5-Tesla (1.5-T) MR scanner closed magnet (Excelart, Vantage, TOSHIBA Medical Systems).

The patients were informed about the nature and duration of the examination (ranged from 15 to $30 \mathrm{~min}$ ) and 
were instructed to remove any metallic objects and not move. Patients were scanned in the prone position with the arm above their head in the so-called Superman position. The wrist was immobilized in neutral position by means of molded holder. The radio carpal joint was used as a landmark. Circular coil was used (C 200) placed over the hand and was wrapped and fixed by rubber bands.

The imaging planes for axial, coronal, and sagittal acquisitions were prescribed with respect to the finger rather than the hand.

An adjacent finger was included within the field of view (FOV) to allow internal comparison.

Whenever we had a surgical final diagnosis, it was considered the gold standard of the results. This would apply to four cases of complete thickness flexor tendon tear that required surgical intervention. MRI was considered the gold standard in the remaining cases.

Protocol of MR imaging The imaging protocol and its parameters (Table 1) consisted of a scout three planes: T1-weighted images (T1WI), axial (T1WI and T2WI), coronal (T1WI, T2WI, proton density with fat saturation and STIR), and sagittal T2WI.

\section{Systematic approach to MRI interpretation}

- Interpretation was done by three of the authors, and all of them have more than three years of clinical expertise in musculoskeletal imaging. The interpretation was done in a conjoint reading team.

- The bones are evaluated for the presence of fracture, osteomyelitis, osteonecrosis, or neoplasm.

- The joints are evaluated for the presence of effusion, synovial proliferation, erosions, osteophytes, and articular cartilage abnormalities.
- Next, the tendons and ligaments are analyzed with special emphasis on the flexor mechanism, extensor mechanism, collateral ligaments, volar plates, and sagittal bands.

- Finally, the surrounding soft tissues are evaluated for the presence of neoplasms, fluid collections, and neurovascular abnormalities.

\section{Sample size and statistical analysis}

- The present study was be done on 42 patients who have clinical suspicion of tendon or ligament lesion of the fingers either in the form of history of trauma, swelling, or painful finger.

- Data were statistically described in terms of mean \pm standard deviation (SD), median and range, or frequencies (number of cases) and percentages when appropriate.

- All statistical calculations and data analysis were done using computer program SPSS (Statistical Package for the Social Science; SPSS Inc., Chicago, IL, USA) release 22 .

- $P$ values less than 0.05 were considered statistically significant.

- Sensitivity, specificity, positive predictive value, negative predictive value and accuracy were calculated by feeding the following formulae in the excel sheet:

- Sensitivity (True Positive Rate)

$$
=\frac{T P}{\text { real positives }}=\frac{T P}{T P+F N}
$$

$$
\text { Specificity }\left(\text { True Negative Rate) }=\frac{T N}{\text { real negatives }}=\frac{T N}{T N+F P}\right.
$$

Table 1 MR imaging parameters used in the study

\begin{tabular}{llll}
\hline Pulse sequences & T1WI & T2WI & STIR \\
\hline TR & $400-600 \mathrm{~ms}$ & $2000-4000 \mathrm{~ms}$ & $2000 \mathrm{~ms}$ \\
TE & $15-25 \mathrm{~ms}$ & $70-100 \mathrm{~ms}$ & $48 \mathrm{~s}$ \\
Slice thickness & $2.5 \mathrm{~mm}$ & $2.5 \mathrm{~mm}$ & $2.5 \mathrm{~mm}$ \\
Interslice gap & $2-3 \mathrm{~mm}$ & $2-3 \mathrm{~mm}$ & $2-3 \mathrm{~mm}$ \\
Matrix size & $256 \times 256$ pixels & $256 \times 256$ pixels & $2-3 \mathrm{~mm}$ \\
FOV & $12 \mathrm{~cm}$ & $12 \mathrm{~cm}$ & $256 \times 256$ pixels \\
Time of examination & $2-3 \mathrm{~min}$ & $2-3 \mathrm{~min}$ & $12 \mathrm{~cm}$ \\
\hline
\end{tabular}

TR; Repetition time, TE; echo time, FOV; field-of-view, STIR; short tau inversion recovery, PD; proton density, msec; millisecond, sec; second, mm; millimeter, cm; centimeter 
Positive Predictive Value (Percision) $=\frac{T P}{\text { Test positive }}=\frac{T P}{T P+F P}$

Negative Predictive Value $=\frac{T N}{\text { Test Negative }}=\frac{T N}{T N+F N}$

Accuracy $=\frac{\text { Test Positive }}{\text { All patients }}=\frac{T P+T N}{T P+F P+T N+F N}$

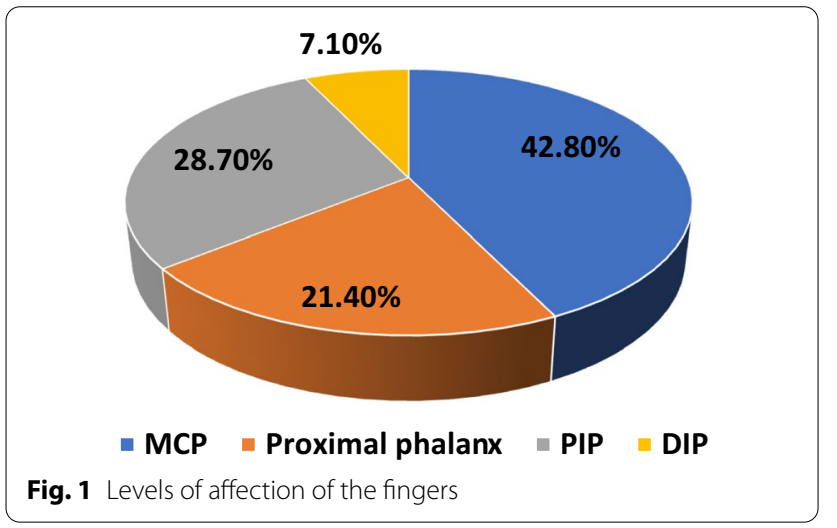

\section{Results}

This prospective study included 42 patients, 33 males, 9 females, with age range from 11 to 56 years (mean age 35 years). All of them had history of trauma.

Striking male affection was noted reaching $78.5 \%$ of the reported cases with history of trauma and subsequent tendon or ligament injury proven by MRI. On the other side, only $21.5 \%$ of the cases were female.

The frequency and percentage according to the side of the injured finger in the study population showed rightsided-hand affection representing $92.9 \%$ and, on the other side, left-sided-hand affection representing 7.1\%

In this study, the most affected finger by trauma was the thumb, which was injured in almost half cases presented with trauma (Fig. 1).

This figure revealed that most common affected finger levels were extending from the MCP till the proximal interphalangeal (PIP) (Fig. 2).

The frequency and percentage according to whether tendon or ligament is affected in patients with history of trauma showed that tendons were affected in $42.9 \%$; meanwhile, ligamentous affection represented $57.2 \%$ of post-traumatic cases (Fig. 3).
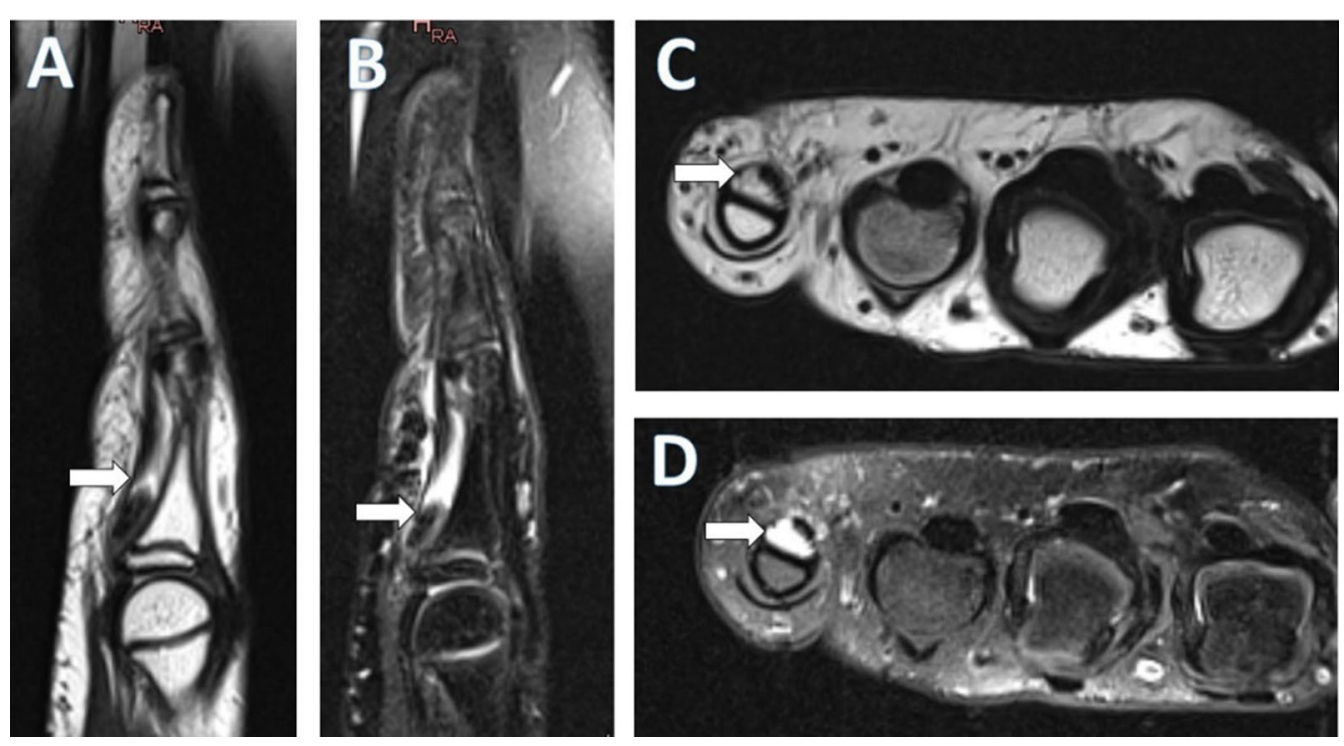

Fig. 2 Male patient 11 years old, complaining of post-traumatic right little finger pain and limitation of flexion. Sagittal T2WI (a), sagittal fat-saturated T2WI (b), axial T2WI (c), and axial PD fat saturated (d) weighted imaging of the right little finger showing complete interruption of the fibers of the flexor digitorum profundus of the right little finger at the level of the proximal phalanx (white arrow) with retraction and fluid filled gap distally. Diagnosis was complete tear of the flexor digitorum tendon of the right little finger with tendon retraction 

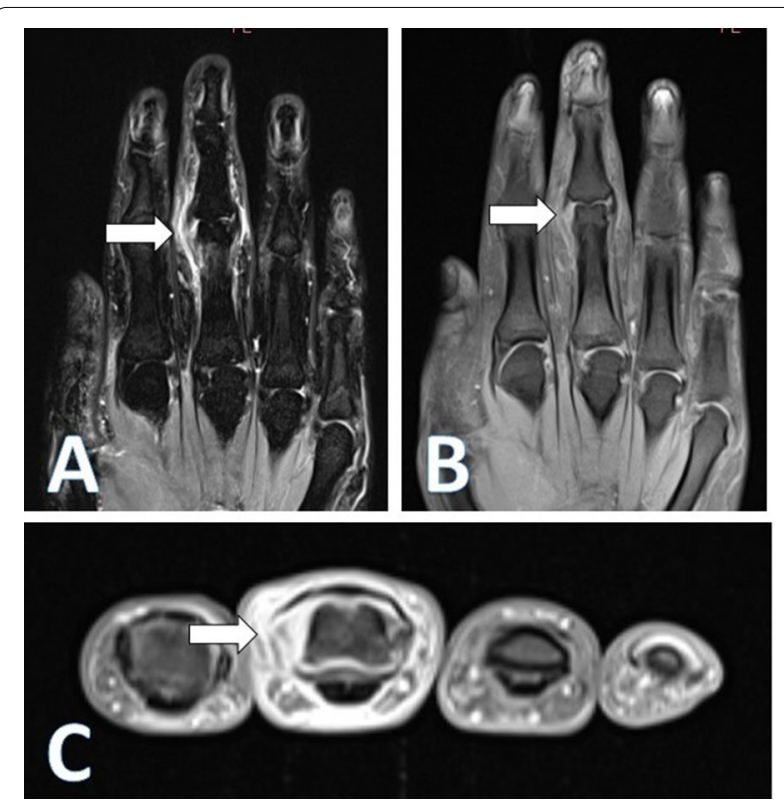

Fig. 3 Male patient 43 years old, complaining of post-traumatic pain at the lateral aspect of the middle finger at the level of the PIP. Coronal T2 STIR (a), coronal T1WI fat-saturated (b), and axial T2Wl fat-saturated (c) imaging of the right middle finger showing discontinuity of the proximal fibers of the lateral collateral ligament of the middle finger at the level of the PIP (white arrow) with related fluid signal intensity. Diagnosis was complete tear of the radial collateral ligament of the right middle finger at the level of the PIP

Table 2 Detection of tendon and ligament tears by MRI in patients with history of trauma:

\begin{tabular}{lrrll}
\hline Affected tendon or ligament & No. & \% Ligament & Total (\%) \\
\hline Flexor tendon & & 12 & 28.6 & 42.9 \\
Extensor tendon & Sagittal band & 3 & 7.15 & \\
& $\begin{array}{l}\text { Extensor appara- } \\
\text { tus lateral band }\end{array}$ & 3 & 7.15 & \\
Ligament & Ulnar collateral & 6 & 14.3 & 57.1 \\
\multirow{2}{*}{ Total } & Radial collateral & 18 & 42.8 & \\
\hline
\end{tabular}

The frequency and percentage according to which tendon was torn in the study population were tabulated (Table 2) and revealed that flexor tendon tear was represented by $28.6 \%$, while extensor tendon tear has occurred in $14.3 \%$ (Fig. 4).

The frequency and percentage of collateral ligament injury in traumatic patients (Table 2) showed that radial collateral injury (of middle finger) was far common than ulnar collateral injury (of thumb) as depicted by MRI (Table 3).

The frequency and percentage according to the type of the tendon tear in the study population (Table 4) showed

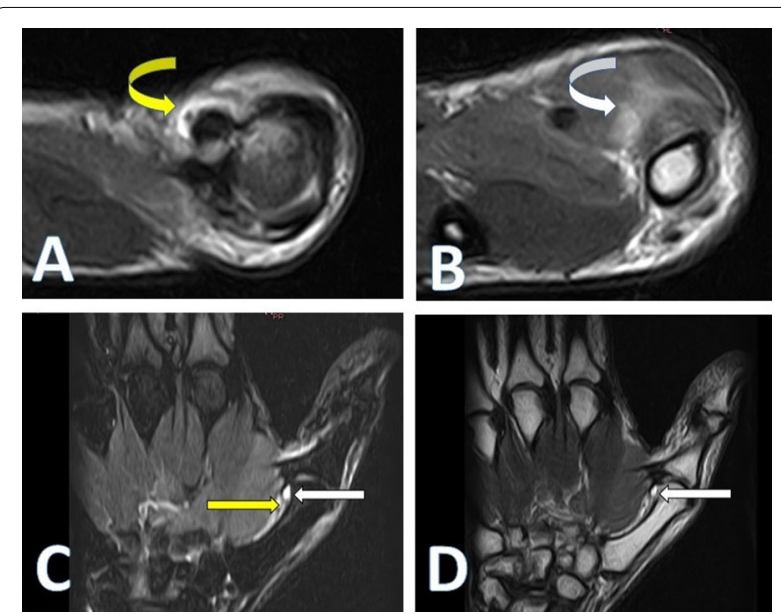

Fig. 4 Male patient 36 years old, complaining of right thumb pain, swelling and limitation of movement following finger trauma. Axial $\mathrm{T} 2 \mathrm{WI}(\mathbf{a}, \mathbf{b})$ of the right thumb revealed fluid signal surrounding the flexor pollicis longus tendon (curved yellow arrow) and edematous changes of the thenar muscles (curved white arrow) and the metacarpophalangeal region. Coronal STIR (c) and coronal proton density (d) weighted imaging of the right thumb at the level of the metacarpophalangeal (MCP) joint showed no definite proximal attachment of the medial collateral ligament (straight yellow arrow) with surrounding hyperintense fluid signal and mild metacarpophalangeal joint effusion (straight white arrow). Diagnosis was right thumb ulnar collateral ligament tear at the level of the metacarpophalangeal joint with tenosynovitis of the flexor pollicis longus and mild metacarpophalangeal joint effusion

Table 3 Distribution of the finger injuries among the fingers and its exact flexor zone as depicted by MRI (affected finger and zone):

\begin{tabular}{llcl}
\hline & Zone & No. & $\%$ \\
\hline Thumb & Zone TI & 1 & 8.3 \\
& Zone TI & 4 & 33.4 \\
Middle & Zone II & 5 & 41.7 \\
Little & Zone II & 2 & 16.6 \\
Total & & 12 & 100 \\
\hline
\end{tabular}

In terms of flexor zones, flexor tendons of zone II and zone T II were the two most affected zones in our study

Table 4 MRI results in detecting thickness of flexor tendon tear in patients with history of trauma

\begin{tabular}{lcc}
\hline Type of tear & MRI & \\
\cline { 2 - 3 } & No. & $\%$ \\
\hline Complete thickness tear & 4 & 33.3 \\
Partial thickness tear & 8 & 66.7 \\
Total & 12 & 100 \\
\hline
\end{tabular}



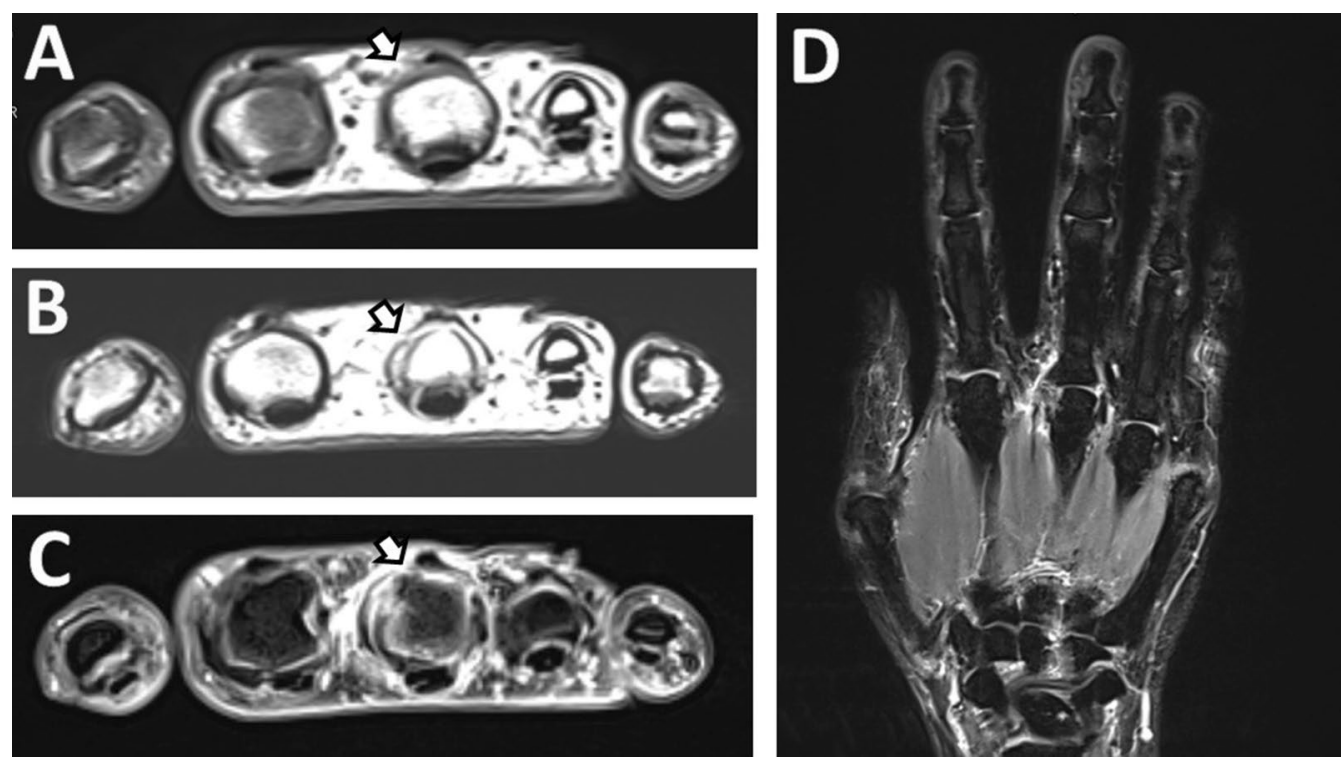

Fig. 5 Female patient 34 years old, complaining of post-traumatic pain over the base of the dorsal aspect middle finger. Axial T1WI (a), axial T2WI (b), axial proton density fat-saturated (c) and coronal STIR (d) weighted imaging of the right middle finger showing partial interruption of the fibers of the radial sagittal band of the middle finger (white arrow) at the level of the metacarpophalangeal (MCP) joint with surrounding edematous changes. Diagnosis was radial sagittal band partial tear of the right middle finger

that complete thickness tear occurred in $33.3 \%$ and partial thickness tear represented by $66.7 \%$ (Figs. 5, 6).

Bone marrow edema and post-traumatic tenosynovitis were noted in six cases representing $14.3 \%$ of cases (Table 5).

\section{Discussion}

Modern magnetic resonance imaging (MRI) techniques and high-quality surface coils can provide exquisite anatomic detail of the small extremities such as the hands and feet. The fine soft tissue contrast resolution provided by MRI allows accurate identification and characterization of a variety of traumatic injuries, arthropathies, and neoplastic processes. Recent advances in gradient hardware, coil design and pulse sequences, as well as the proliferation of $3 \mathrm{~T}(3 \mathrm{~T})$ MR units, allow very small field-of-view imaging at high resolution. The fine soft tissue contrast resolution of MRI allows assessment of the tendons, ligaments, and intricate pulley systems of the fingers. Normal tendons and ligaments of the hands typically demonstrate low signal intensity on MR imaging. In contrast, these structures exhibit increased signal intensity or abnormal morphology when injured [12].

This study included 42 patients, 33 males, 9 females, with age range from 11 to 56 years (mean age 35 years). All of them had history of trauma. This agreed with the study of De Jong et al. [13], which showed that mean age at the time of injury was 35.9 years (range 1-91 years).
This corresponds to the age of the workforce most likely to have more physical labor-intensive occupations, which may place them at increased risk for injury.

The present study showed marked right-sided-hand affection $(92.9 \%)$, while, on the other side, left-sidedhand affection represented by $7.1 \%$ only. This agreed with the study of De Jong et al. [13] that found that right-hand finger affection is about $91.4 \%$, while the left-hand fingers were affected in only about $7.6 \%$ of cases.

In this study, striking male affection was noted reaching almost $78.5 \%$ of the reported cases with history of trauma and subsequent tendon or ligament injury proven by MRI. In contrast, only $21.5 \%$ of the cases were female. This agreed with the study of Kringstad et al. [14], which announced that males sustained more wounds from using hazardous equipment and thus have more severe injuries. Males have a significantly higher incidence of traumatic tendon injuries to the hand or wrist compared to females, a finding in accordance with previous studies. This may be partly due to the nature of gender-related professional work and recreational activities.

Our study showed that MRI can detect the exact site and level of tendon and ligament affection, which played a major role in correct surgical advancement and proper choice of the incision site. This agreed with the study of Jacob [15], which announced that advanced imaging with MRI is helpful to both confirm the diagnosis and accurately define the exact location of tendon injury for surgical planning. 

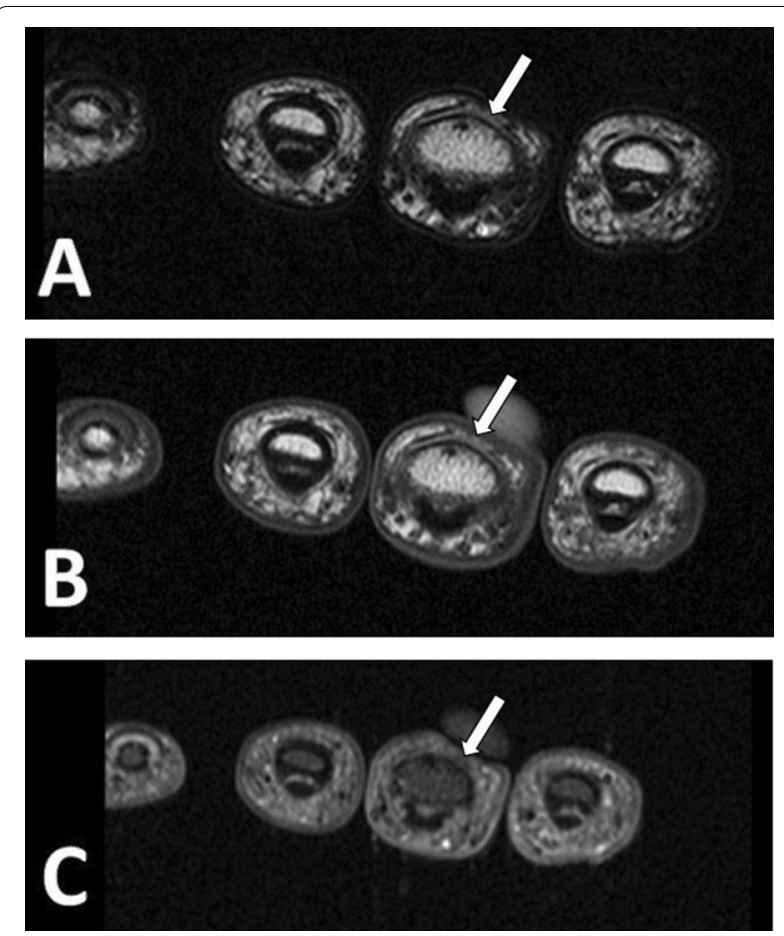

Fig. 6 Male patient 28 years old, complaining of weakness in extension of the middle finger of the right hand with remote history of hand trauma. Axial T2WI (a), axial T1WI (b) and axial STIR (c) weighted imaging of the right middle finger at the level of the proximal interphalangeal joint (PIP) showed abnormally thinned out lateral band of the extensor apparatus of the middle finger (white arrow) with preserved thickness of the medial band of the similar apparatus. Diagnosis partial tear of the lateral band of the extensor apparatus of the right middle finger

Table 5 Accuracy analysis of MRI compared to surgery in detection of complete tear of the flexor tendons

\begin{tabular}{ll}
\hline & MRI \\
\hline True positive & 4 \\
True negative & 0 \\
False positive & 0 \\
False negative & 0 \\
Sensitivity & $100 \%$ \\
Positive predictive value (PPV) & $100 \%$ \\
Negative predictive value (NPV) & $0 \%$ \\
Accuracy & $100 \%$ \\
\hline
\end{tabular}

The MRI results compared with surgery results with $100 \%$ accuracy of MRI in detection of complete tear of flexor tendons

The present study showed that in terms of finger affection, the middle finger and the thumb were the most injured fingers, $50 \%$ and $35.7 \%$, respectively. This agreed with the study of De Jong et al. [13] that revealed marked affection of the thumb, index and middle finger in the study population, collectively about $68 \%$ of injured fingers.

This study showed that regarding the frequency and percentage of affection of the tendon or ligament in patients with history of trauma, ligamentous affection represented $57.1 \%$ of post-traumatic cases; meanwhile, tendons were affected in $42.9 \%$. This agreed with the results of the study done by Woo et al. [16] who found that in the 50 patients with finger injuries, there were 33 cases of ligament injuries and 17 cases with tendon injuries.

Our study showed more affection of the flexor tendons (28.6\%) compared to extensor tendons (14.3) as depicted by MRI. This was disagreeing with the results of the study of De Jong et al. [13] that showed extensor tendon injuries accounted for more injuries than flexor tendon injuries. In total, 395 extensor tendons and 297 flexor tendons of the hand were injured in that study. Extensor tendon injuries in the extensor zones of the fingers (namely zones I, II, III, IV, TI, TII and TIII) were about $33.4 \%$ of extensor tendon injuries of the hand, while injured cases at the flexor zones of the fingers (namely zones I, II, TI, TII) constituted about $27.6 \%$ of flexor tendon injuries of the hand. This may be attributed to his large study population.

This study showed that, in terms of flexor zones, flexor tendons of the fingers other than thumb showed affection of zone II (middle finger affected in 5 cases and little finger affection in 2 cases). Tendon retraction with gapping was noted in the affected flexor tendon of the little finger. Flexor tendons of the thumb were dominantly affected at zone T II ( 4 cases) and only 1 case at zone T I (at the level of distal portion of proximal phalanx). This agreed with the study of De Jong et al. [13] that found that of all flexor tendon zones, zone two was significantly more often than every other flexor zone. This was followed by zone I and zone T II.

The present study showed that the frequency and percentage of complete thickness tendon tear, as depicted by MRI, is represented by $33 \%$ and partial thickness tendon tear represented by $67 \%$. This partially agreed with the 10-year study done by De Jong et al. [13] that involved 458 cases of acute traumatic tendon injuries of the hand with injuries affecting 692 tendons, of which 184 (26.6\%) were reported to be only partially lacerated. This modest difference is related to the difference of the study population, as de Jong excluded the patient with closed tendon injury.

This study showed that MRI detection of the type of tendon tear is represented by $100 \%$ of the traumatic cases. This also almost agreeing with Gupta et al. [17], who reported that MRI allows diagnosis of complete or partial tendon tears, helps determine the location of 
the tears and the degree of tendon retraction, and helps exclude any associated avulsion fractures or other injuries. For these reasons, MRI is often used to classify and optimally manage these injuries.

In our study, the frequency and percentage of collateral ligament injury showed that radial collateral injury (of middle finger) was far common than ulnar collateral injury (of thumb) as depicted by MRI. Disruption of the proximal attachment of the radial collateral ligament of the middle finger with fluid signal fills the gap. Another case showed no definite proximal attachment of the medial collateral ligament of the thumb finger with replacement by fluid signal and associated mild metacarpophalangeal joint effusion. This agreed with Kadow and Fowler [7], who stated that MR imaging criteria for diagnosis of acute collateral ligament tears include discontinuity, detachment, or thickening of the ligament together with increased intraligamentous signal intensity on T2-weighted images, which is indicative of edema or hemorrhage. This also agreed with Gupta et al. [17], who reported that collateral ligament tears are typically seen at their proximal attachment. Forced ulnar deviation at the metacarpophalangeal joint may result in isolated tears of the radial collateral ligament. Isolated tears of the ulnar collateral ligaments at the metacarpophalangeal joint are relatively rare.

In the present study, MRI can detect bone marrow edema that was present in about $26.6 \%$ of the traumatic patients. This agreed with the study of Roemer et al. [18] that reported that subchondral bone marrow edema-like signal alterations exhibit typical signal characteristics on MRI and are common but nonspecific findings. MRI is the ideal tool to assess these pathologies as the subchondral bone marrow cannot be visualized by X-ray or ultrasound technology.

In our study, extensor system affection was noted in $14.3 \%$ of affected cases. Radial sagittal band affection with surrounding soft tissue edematous changes was noted. Also, lateral band of the extensor apparatus showed thinning out notably in T1WI denoting partial tear. This agreed with the study of Young et al. [19] that reported that injury of the radial sagittal band may result in ulnar subluxation of the extensor tendon. The degree of tendon instability is determined by the extent of sagittal band disruption. In a partial tear, a proximal tear rather than a distal tear of the sagittal band contributes to instability. In a complete tear, there is dislocation of the extensor tendon into the intermetacarpal space.

\section{Conclusions}

In conclusion, MRI is an essential technique to obtain a correct pre-surgical diagnosis. It is of utmost importance to possess an in-depth knowledge of finger radiological anatomy in detail, as well as the appearance of the different pathologic entities on MRI. It provides a great assessment of the tendon and ligaments tear whether partial or complete, any associated marrow edema or bony fragment avulsion and the extent of retraction in cases of complete tear.

\section{Recommendations}

Larger studies using MRI should be performed to allow exposure to larger numbers of extensor tendon injuries. Using MRI three-dimensional acquisition techniques and a higher Tesla rate might better define the course and integrity of tendons allowing images in the longitudinal and oblique planes. Also, assessing the tendons and ligaments using dynamic MRI would provide better assessment of partial injuries and its impact upon the dynamics of the finger joints.

\section{Abbreviations}

MRI: Magnetic resonance imaging; MR: Magnetic resonance; FOV: Field of view; T1Wl: T1-weighted image; T2Wl: T2-weighted image; STIR: Short tau inversion recovery; PD: Proton density; TR: Repetition time; TE: Echo time; SPSS: Statistical Package for the Social Science; MCP: Metacarpophalangeal; PIP: Proximal interphalangeal; DIP: Distal interphalangeal; IV: Intravenous.

\section{Acknowledgements}

None.

Authors' contributions

All authors have contributed equally to this study. All authors read and approved the final manuscript.

Funding

The authors declare that the current study had no funding from any resource.

\section{Availability of data and material}

The authors confirm that all data supporting the findings of the current study are available within the article. Also, all the datasets used during the current study are available from the corresponding author on a reasonable request.

\section{Declarations}

\section{Ethics approval and consent to participate}

All study procedures will be carried out and approved by the Ethical Committee of Menoufia Faculty of Medicine. Consent: All participants in this study received a detailed explanation about the aim, objectives, and methodology of the study before enrollment. Also, all participants gave their written consent to participate in the study.

\section{Consent for publication}

All subjects included in the current research gave their written informed consent to publish the data contained within the study.

\section{Competing interests}

The authors declare that they have no competing interests.

\section{Author details}

${ }^{1}$ Faculty of Medicine, Menoufia University, Shbein Elkom, Menoufia Governorate, Egypt. ${ }^{2}$ Faculty of Medicine, Menoufia University, shebin el kom, Menoufia Governorate, Egypt. ${ }^{3}$ Faculty of Medicine, Menoufia University, shebin el kom, Menoufia, Egypt. 
Received: 11 August 2021 Accepted: 19 October 2021

Published online: 17 November 2021

\section{References}

1. Li YX, Ye W, Bai RJ, Zhan HL, Liu Y, Qian ZH (2017) MRI of the finger ligament and tendon injuries: imaging technique and clinical application. Zhonghua Yi Xue Za Zhi 97(11):847-851

2. Meraj S, Gyftopoulos S, Nellans K, Walz D, Brown MS (2017) MRI of the extensor tendons of the wrist. Am J Roentgenol 209(5):1093-1102

3. Pilania K, Jankharia B (2018) A pictorial essay on focused magnetic resonance imaging of the normal anatomy and various injuries of the finger. Can Assoc Radiol J 69(4):437-449

4. Tang JB, Lalonde D (2020) Surgery management of flexor tendon injuries. Rehabilitation of the Hand and Upper Extremity, E-Book, p 402

5. Chauhan A, Jacobs B, Andoga A, Baratz ME (2014) Extensor tendon injuries in athletes. Sports Med Arthrosc Rev 22(1):45-55

6. Colzani G, Tos P, Battiston B, Merolla G, Porcellini G, Artiaco S (2016) Traumatic extensor tendon injuries to the hand: clinical anatomy, biomechanics, and surgical procedure review. J Hand Microsurg 8(1):2

7. Kadow TR, Fowler JR (2017) Thumb injuries in athletes. Hand Clin 33(1):161-173

8. Arthozoul M, Brun C, Laffosse JM, Martinel V, Grolleau JL, André A (2015) Avulsion of flexor digitorum profundus secondary to enchondroma of the distal phalanx. Case report and literature review. Chir Main 34(4):210-214

9. King EA, Lien JR (2017) Flexor tendon pulley injuries in rock climbers. Hand Clin 33(1):141-148

10. Makkouk AH, Oetgen ME, Swigart CR, Dodds SD (2008) Trigger finger: etiology, evaluation, and treatment. Curr Rev Musculoskelet Med 1(2):92-96

11. Heiss R, Librimir A, Lutter C, Janka R, Kuerten S, Roemer FW, Nagel AM, Uder M, Bayer T (2021) MRI of finger pulleys at 7T—direct characterization of pulley ruptures in an ex vivo model. Diagnostics 11(7):1206

12. Malone JC, Preston KS, Garner HW, Bestic JM, Wessell DE, Peterson J J (2016) MRI of the hand and fingers. Appl Radiol 45(2):9-21

13. de Jong JP, Nguyen Jesse T, Sonnema AJ, M, Nguyen Emily C, Amadio Peter C, Moran Steven L (2014) The incidence of acute traumatic tendon injuries in the hand and wrist: a 10-year population-based study. Clin Orthop Surg 6(2):196-202

14. Kringstad O, Dahlin LB, Rosberg HE (2019) Hand injuries in an older population-a retrospective cohort study from a single hand surgery centre. BMC Musculoskelet Disord 20(1):1-2

15. Almusa E, Peterson WM, Bianchi S, Jacob D, Hoffman D (2013) Radiological investigations. In: Chick G (ed) Acute and chronic finger injuries in ball sports. Paris, Springer, pp 89-124

16. Woo SH, Lee YK, Kim JM, Cheon HJ, Chung WH (2017) Hand and wrist injuries in golfers and their treatment. Hand Clin 33(1):81-96

17. Gupta P, Lenchik L, Wuertzer SD, Pacholke DA (2015) High-resolution 3-T MRI of the fingers: review of anatomy and common tendon and ligament injuries. Am J Roentgenol 204(3):W314-W323

18. Roemer FW, Neogi T, Nevitt MC, Felson DT, Zhu Y, Zhang Y, Lynch JA, Javaid MK, Crema MD, Torner J, Lewis CE (2010) Subchondral bone marrow lesions are highly associated with, and predict subchondral bone attrition longitudinally: the MOST study. Osteoarthritis Cartilage 18(1):47-53

19. Young CM, Rayan GM (2000) The sagittal band: anatomic and biomechanical study. J Hand Surg [Am] 25:1107-1113

\section{Publisher's Note}

Springer Nature remains neutral with regard to jurisdictional claims in published maps and institutional affiliations.

\section{Submit your manuscript to a SpringerOpen ${ }^{\circ}$ journal and benefit from:}

- Convenient online submission

- Rigorous peer review

- Open access: articles freely available online

- High visibility within the field

- Retaining the copyright to your article

Submit your next manuscript at $\boldsymbol{\nabla}$ springeropen.com 